\title{
Lewy Body Dementia Association's Research Centers of Excellence Program: Inaugural Meeting Proceedings
}

Bethany Peterson ${ }^{1}$, Melissa Armstrong ${ }^{2}$, Douglas Galasko ${ }^{3}$, James E. Galvin ${ }^{4}$, Jennifer Goldman ${ }^{5}$, David Irwin ${ }^{6}$, Henry Paulson ${ }^{7}$, Daniel Kaufer ${ }^{8}$, James Leverenz ${ }^{9}$, Angela Lunde ${ }^{10}$, lan G. McKeith ${ }^{11}$, Andrew Siderowf ${ }^{6}$, Angela Taylor ${ }^{1}$, Katherine Amodeo ${ }^{12}$, Matt Barrett ${ }^{13}$, Kimiko Domoto-Reilly ${ }^{14}$, John Duda ${ }^{6}$, Stephen Gomperts ${ }^{15}$, Neill Graff-Radford ${ }^{16}$, Samantha Holden ${ }^{17}$, Lawrence Honig ${ }^{18}$, Daniel Huddleston ${ }^{19}$, Carol Lippa ${ }^{20}$, Irene Litvan ${ }^{3}$, Carol Manning ${ }^{13}$, Karen Marder ${ }^{18}$, Charbel Moussa ${ }^{21}$, Chiadi Onyike ${ }^{22}$, Fernando Pagan ${ }^{21}$, Alexander Pantelyat ${ }^{22}$, Victoria Pelak ${ }^{17}$, Kathleen Poston ${ }^{23}$, Joseph Quinn ${ }^{24}$, Irene Richard ${ }^{12}$, Liana S. Rosenthal ${ }^{22}$, Marwan Sabbagh ${ }^{25}$, Douglas Scharre ${ }^{26}$, Sharon Sha ${ }^{23}$, Holly Shill' ${ }^{27}$, Yasar Torres-Yaghi ${ }^{21}$, Tina Christie ${ }^{1}$, Todd Graham ${ }^{1}$, lan Richards ${ }^{1}$, Mike Koehler ${ }^{1}$ and Brad Boeve ${ }^{10^{*}}$ (D)

\begin{abstract}
The first Lewy Body Dementia Association (LBDA) Research Centers of Excellence (RCOE) Investigator's meeting was held on December 14, 2017, in New Orleans. The program was established to increase patient access to clinical experts on Lewy body dementia (LBD), which includes dementia with Lewy bodies (DLB) and Parkinson's disease dementia (PDD), and to create a clinical trials-ready network. Four working groups (WG) were created to pursue the LBDA RCOE aims: (1) increase access to high-quality clinical care, (2) increase access to support for people living with LBD and their caregivers, (3) increase knowledge of LBD among medical and allied (or other) professionals, and (4) create infrastructure for a clinical trials-ready network as well as resources to advance the study of new therapeutics.
\end{abstract}

Keywords: Lewy body dementia, Lewy Body Dementia Association, Parkinson's disease dementia

\section{Introduction}

The first Lewy Body Dementia Association (LBDA) Research Centers of Excellence (RCOE) Investigator's meeting was held on December 14, 2017, in New Orleans. The program was established to increase patient access to clinical experts on Lewy body dementia (LBD), which includes dementia with Lewy bodies (DLB) and Parkinson's disease dementia (PDD), and to create a clinical trials-ready network. Mayo Clinic, Rochester $\mathrm{MN}$, was designated the coordinating center following a competitive application process. The 24 centers were selected by an application and peer-review process.

\footnotetext{
* Correspondence: bboeve@mayo.edu

${ }^{10}$ Mayo Clinic campus, 200 1st Street SW, Rochester, MN 55905, USA

Full list of author information is available at the end of the article
}

\section{Background}

The top national research priority for LBD is to initiate clinical trials to address symptoms that have the greatest impact on patient function and caregiver burden. LBDA's RCOE program establishes infrastructure to run multi-center studies with expertise, centralization, and standardized data collection. The program will yield a well-characterized, nationwide group of LBD patients for rapid recruitment into therapeutic trials.

\section{Aims of the program}

The major aims are to:

- Increase access to high-quality clinical care

- Increase access to support for people living with LBD and their caregivers

(C) The Author(s). 2019 Open Access This article is distributed under the terms of the Creative Commons Attribution 4.0 International License (http://creativecommons.org/licenses/by/4.0/), which permits unrestricted use, distribution, and reproduction in any medium, provided you give appropriate credit to the original author(s) and the source, provide a link to the Creative Commons license, and indicate if changes were made. The Creative Commons Public Domain Dedication waiver (http://creativecommons.org/publicdomain/zero/1.0/) applies to the data made available in this article, unless otherwise stated. 
- Increase knowledge of LBD among medical and allied (or other) professionals

- Create infrastructure for a clinical trials-ready network as well as resources to advance the study of new therapeutics.

Four working groups (WG) were created to pursue the LBDA RCOE aims. WG chairs (JEG, JGG, HP, MJA, DK, DI, JBL, AS) were selected; all PIs self-selected in which WGs they would participate. Each WG had several goals to achieve for the meeting, summarized below.

\section{Working groups}

\section{Clinical care and professional education WG}

WG goals are to ascertain best practice clinical guidelines and identify opportunities for professional LBD education. The group discussed strategies to solicit feedback from experts and other stakeholders on diagnosis and symptom management. Diagnosis using the fourth consensus report of the DLB consortium was emphasized [1], but challenges regarding its widespread implementation remain. Important clinical milestones in LBD management include referrals to allied health therapies, utilization of community resources, and entry into skilled nursing and long-term care facilities. Examples of LBD research and clinical service toolkit development such as the DIAMOND-Lewy research program may help inform the LBDA RCOE efforts [2].

Increasing awareness and disseminating knowledge on LBD to healthcare professionals remains a critical priority. Key target audiences include (a) healthcare professionals such as physicians, advanced practice providers, and trainees practicing in Neurology, Psychiatry, Geriatric Psychiatry, Geriatrics, Sleep Medicine, Cardiology, Urology, Gastroenterology, and Palliative Care; those practicing in Psychology and Neuropsychology; and those practicing in long-term care settings; (b) allied health professionals (nurses, social work, physical, occupational and speech therapy, nutrition, arts/music therapy, chaplaincy, and hospice); and (c) healthcare workers in specific environments, including hospitals, assisted living facilities, long-term care facilities, and adult day programs.

\section{Community education and support WG}

WG goals are to develop core themes and materials, and identify opportunities for community education, assistance for support groups, and best practices for caregiver education and support for non-pharmacological approaches to behavioral changes in LBD.

The WG surveyed 23 RCOE sites. Ten sites (43\%) already hosted LBD-specific support groups, most commonly targeting caregivers alone or caregivers and people with LBD together. These groups were typically led by either clinical staff or former caregivers and met on a monthly basis. Sites preferred either in-person or mixed in-person/virtual monthly support groups led by clinical staff. Most sites favored targeting caregivers and/ or early-stage people with LBD; some sites suggested support groups for moderate- and late-stage people with LBD. Trained facilitators were described as essential.

LBDA RCOE sites commonly educate people with LBD and their families with LBDA-produced materials, the LBD booklet from the National Institutes of Health, and materials produced by the individual sites.

\section{Clinical trial design and optimization WG}

WG goals are to identify ongoing or planned therapeutic trials in LBD, identify and address key gaps in clinical assessment tools for use in diagnosis of LBD and clinical trials, identify current or emerging biomarkers relevant for LBD clinical trials, and develop strategies for implementation. Collectively, the LBDA RCOE network is estimated to see $>2600$ new patient visits $(\sim 1300$ DLB and $\sim 1300$ PDD) and $>7,500$ return visits $(\sim 3000$ DLB and $\sim 4500$ PDD) annually. The majority of sites are very active in clinical trials for LBD and related disorders, with 16 centers participating in $\geq 1$ clinical trial for LBD and collectively enrolling $\sim 430$ LBD patients in the past year. Furthermore, 21 sites participated in $\geq 2$ and 18 sites are active in $\geq 5$ clinical trials for related neurodegenerative disorders.

The RCOE investigator survey and discussion revealed a strong interest in trials focused on both symptomatic therapies and potential disease-modifying agents. Prioritized targets for non-pharmacological interventions included caregiver-stress, agitation, and motor function limitations. One critical need is to identify and develop clinically meaningful DLB clinical outcome measures, including ways to account for and/or quantify the effects of cognitive fluctuations. Emerging biomarkers were classified on their potential for diagnostic and/or prognostic use based on review of the current state of the science. High-yield applications of diagnostic biomarkers for LBD included identifying prodromal disease, differentiation of LBD from other dementias, and stratification of biologically meaningful subgroups of DLB patients such as those with significant AD co-pathology or those harboring a glucocerebrosidase gene mutation. There was strong interest in the need for alpha-synuclein-specific markers; however, it was felt that current assays require further development and validation. Other top priorities identified included tissue-validation and standardization of imaging and fluid biomarkers, and the development of an infrastructure for sharing biomarker specimens and data that will be obtained in future clinical trials to the research community at large. 


\section{Industry engagement WG}

WG goals are to determine general principles for interacting with industry partners and determine requirements for industry-sponsored clinical trials, including data and sample sharing and publication plan, contract and budget development, and protocol optimization. The WG serves as a central resource for exploring collaborations between the RCOE and industry on LBD clinical trials.

To maximize efficiencies in collaborations with industry, this working group has proposed an accessible, centralized data resource on the clinical trial capabilities of all RCOE sites. This will facilitate exploring clinical research collaborations and expedite site selection for industry-sponsored trials. Each site will determine its unique clinical trial capacity, its ability to recruit people with LBD for trial enrollment, and its testing capabilities. The RCOE program will strive to use a standardized contract and a central institutional review board. In parallel with the effort to build the infrastructure, this working group will provide guidance on establishing expert panels to advise clinical trial sponsors.

\section{Conclusions}

The LBDA established the RCOE program to accelerate access to high-quality care and support, increase disease awareness among healthcare providers, and advance the study of symptomatic and disease-modifying treatments. As the RCOE program grows and evolves, it will continue to share its successes and lessons learned with the scientific community. The next RCOE Principal Investigator's meeting will be in June 2019.

\section{Abbreviations}

AD: Alzheimer's disease; DLB: Dementia with Lewy bodies; LBD: Lewy body dementia; LBDA: Lewy Body Dementia Association; PDD: Parkinson's disease dementia; RCOE: Research Centers of Excellence; WG: Working group

\section{Acknowledgements}

We extend our appreciation to the staff of all centers in the LBDA RCOE program, and particularly to our patients and their families for whom we serve.

\section{Funding}

This work is supported by the Lewy Body Dementia Association.

\section{Authors' contributions}

All authors contributed to the concept and design on the RCOE program The chairs of the working groups (JEG, JGG, HP, MJA, DK, DI, JBL, AS) authored their sections, and all authors edited and contributed to the data collection. All authors read and approved the final manuscript.

\section{Competing interests}

Melissa Armstrong-Dr. Armstrong receives research support from ARHQ (K08HS24159), a 1Florida ADRC pilot grant, as the local PI of a Lewy Body Dementia Association Research Centers of Excellence, and from the University of Florida Dorothy Mangurian Headquarters for Lewy Body Dementia and the Raymond E. Kassar Research Fund for Lewy Body Dementia. She receives compensation from the American Academy of Neurology for work as an evidence-based medicine methodology consultant and serves on the level of evidence editorial board for Neurology and related publications (uncompensated). She receives royalties from the publication of the book Parkinson's Disease: Improving Patient Care (2014), and she has received honoraria for presenting at American Academy of Neurology annual meetings and participating in Medscape CME.

Douglas Galasko has received consulting fees from vTv Pharmaceuticals, Inc., and for serving on the Data Safety Monitoring Boards for Cognition

Therapeutics and for Proclara Biosciences. He has received compensation for serving as editor for Alzheimer's Research \& Therapy. He has received clinical trial funding from Eli Lilly, Biogen, and Roche/Genentech and research funding from the National Institutes of Health $(\mathrm{NIH})$ and the Michael J. Fox Foundation. James E Galvin has received grant support from NIH, Michael J Fox Foundation, Harry T. Mangurian Foundation, Lewy Body Dementia Association, and the Albert Charitable Trust and has received support for clinical trials from Biogen and Novartis. He has received consulting fees from Biogen, Axovant, Roche, Eisai, Lilly, Bracket, and Medavante and has licensing agreements with Roche, Lilly, Biogen, Quintiles, Roobrik, Continuum Clinical, and Langland.

Jennifer Goldman has received grant/research support from NIH, Michael J. Fox Foundation, Parkinson Foundation, CHDI (Enroll-HD, site-PI), Rush University, Acadia, and Biotie/Accorda (site-PI); consulting fees from Acadia Aptinyx, and Sunovion; and honoraria from the International Parkinson and Movement Disorder Society, American Academy of Neurology.

David Irwin receives support from NIH, Brightfocus Foundation, Cure PSP, and Penn Institute on Aging.

Henry Paulson has received research funding from $\mathrm{NIH}$ and with lonis Pharmaceuticals.

Daniel Kaufer has served as an investigator for clinical trials sponsored by Abbvie, Axovant, Janssen Research \& Development, Navidea

Biopharmaceuticals, and TauRx. He has consulted for Abbvie, Axovant, Janssen Research \& Development, and Takeda/Zinfandel. He receives research funding from the NIH, HRSA, and Bryan Family Foundation.

James Leverenz has served as an investigator for clinical trials sponsored by Avid and Biogen. He serves as a consultant for Aptinyx, Eisai, Sanofi, and Takeda. He receives grant support from the Alzheimer's Association, Department of Defense, Lewy Body Dementia Association, Michael J Fox Foundation, NIH, Alzheimer's Drug Discovery Foundation, and GE Healthcare. Angela Lunde receives NIH support in her role as co-director of the Outreach, Recruitment, and Engagement Core.

Ian G McKeith has advised on clinical trials for Axovant, Eisai, Sumitomo Dainippon Pharma, and Sanofi.

Andrew Siderowf has been a consultant to the following companies in the past year: Biogen, Voyager Therapeutics, Merck, Denali, Wave Life Sciences, and Prilenia Therapeutics. He has received grant funding from the Michael J. Fox Foundation and $\mathrm{NIH}$.

Angela Taylor is an employee of the Lewy Body Dementia Association. Katherine Amodeo has received funding from the Michael J Fox Foundation Edmond J. Safra Fellowship in Movement Disorders. She serves as an investigator for a clinical trial sponsored by Roche.

Matt Barrett has served as an investigator for clinical trials sponsored by the $\mathrm{NIH}$, Azevan, Axovant, Merck, Eisai, Biogen, and Acadia. He receives research support from the Department of Defense.

Kimiko Domoto-Reilly has served as an investigator for clinical trials sponsored by Avid Radiopharmaceuticals, Biogen, and Janssen Pharmaceuticals. She has served as Advisory Board consultant for Biogen. She receives research support from $\mathrm{NIH}$.

John Duda declares that he has no competing interests.

Stephen Gomperts serves on the Advisory Board of Acadia Pharmaceuticals. He receives research support from the National Institutes of Health and the Department of Defense.

Neill Graff-Radford serves as an investigator on clinical trials for Novartis, Biogen, Lilly, and AbbVie. He has several NIH-funded studies and is the Mayo Clinic David Eisenberg Professor. He receives royalties from a chapter for UpToDate on Normal Pressure Hydrocephalus.

Samantha Holden has received research support from the Michael J. Fox Foundation for Parkinson's Research and honoraria from the Parkinson Association of the Rockies.

Lawrence Honig has received funding as a consultant for Eisai and Miller Communications and has received funding as a study investigator for AbbVie, Biogen, Bristol-Myers Squibb, Eisai, Eli Lilly, Genentech, Lundbeck, F. Hoffmann-La Roche, and vTv.

Daniel Huddleston receives research support from the NIH (1K23NS10594401A1, 1R34AG056639-10A1), the Georgia Research Alliance, the Michael J. Fox Foundation (MJF-10854), the American Parkinson's Disease Foundation, 
and the Lewy Body Dementia Association. He is an inventor on one issued patent (USPTO\# 9600881) for MRI acquisition and processing methods and one provisional patent application for MRI analysis tools.

Carol Lippa has served as an investigator for clinical trials sponsored by Lilly, Roche/Genentech and Avanir. She serves on the Scientific Advisory Board of the AFTD and LBDA, and the Delaware Valley chapter of the Alzheimer's Association. She receives research support from NIH. She also owns index funds that may include healthcare companies.

Irene Litvan is supported by NIH grants: 5P50 AG005131-31, 5T35HL007491, and 1U54NS092089-01; Parkinson Study Group, Michael J Fox Foundation, AVID Pharmaceuticals, Abbvie, Biogen, and Roche. She receives her salary from the University of California San Diego. She has been a member of the Abbvie and Biogen Advisory Boards and was a member of the Biotie/ Parkinson Study Group Medical Advisory Board. She receives honorarium as Chief Editor of Frontiers in Neurology.

Carol Manning has served as an investigator for clinical trials sponsored by Axovant, Roche, Eisai, Janssen, and Axsome. She currently receives support from the Administration for Community Living.

Karen Marder has served as a site investigator for TEVA, Azevan, and Vaccinex. She receives royalties as a section editor for Springer Publishing and grant support from NIH, The Parkinson Foundation, Michael J. Fox, HDSA, CHDI, and LBDA.

Charbel Moussa serves as an investigator on Nilotinib trials in Parkinson's diseases, Alzheimer's disease, and Huntington's disease. He also serves as an investigator on Nilotinib and Bosutinib in Lewy Body dementia and receives licensing fees from Axovant sciences on licenses to treat neurodegenerative diseases with tyrosine kinase inhibitors. He receives funding from the NIH, Alzheimer's Association, Alzheimer's Drug Discovery Foundation, Michael J. Fox Foundation, Novartis, Sun Pharmaceuticals Research Corporation (SAPRC), Muccopolysacharoidoisis Society, and the Emerald Foundation Inc. Dr. Moussa also served on Advisory Boards for Osmolex/Osmotica, GE Heathcare, SPARC, UCB Sciences and MemoFit. Dr. Moussa received philanthropy from patients and other philanthropic organization for clinical trials. Dr. Moussa is listed as an investigator on several Georgetown University patents to treat neurodegenerative diseases.

Chiadi U. ONYIKE receives research funding from the $\mathrm{NIH}$, the $\mathrm{CIHR}$, and Biogen, Inc. He is also supported by the Jane Tanger Black Fund for YoungOnset Dementias, the Nancy H. Hall Fund for Geriatric Psychiatry, and the gift from Joseph Trovato.

Fernando Pagan receives educational support from Medtronic, research support from US World Meds, and consults for Abbvie, Acadia, Accorda, Adamas, Merz,Neurocrine, Sunovion, Teva, and US World Meds.

Alexander Pantelyat has served as an investigator for a clinical trial sponsored by Biogen. He serves on the CurePSP Centers of Care steering committee. He receives research support from $\mathrm{NIH}$ and the International Parkinson Disease and Movement Disorders Society.

Victoria Pelak is serving as an investigator and/or co-investigator for clinical trials sponsored by Eli Lilly, GenSightBiologics, and Quark Pharmaceuticals. She receives royalties from the publication of a book entitled Neuroophthalmology: Diagnosis and Management (Elsevier, 3rd Edition) and for online book chapters (Visual Hallucinations and Ocular Myasthenia Gravis) from Up-To-Date, Inc. She receives research support from NIH, the Alzheimer's Association, and the North American Neuro-ophthalmology Society. Kathleen Poston has served as an investigator for clinical trials sponsored by AstraZeneca and Sanofi. She serves as a consultant for Allergan. She serves on the Steering Committee for the Parkinson's Progression Markers Initiative. She receives research support from the NIH and the Michael J Fox Foundation for Parkinson's disease Research.

Joseph Quinn has served as an investigator for clinical trials for Prothena, Sanofi, and Abbvie and has received compensation for serving on a DSMB for vTV Pharmaceuticals. He receives research support from the NIH, VA, and DOD. Irene Richard has received research grant support from the Michael J. Fox Foundation, National Institutes of Health, and F. Hoffmann-La Roche and Ltd. and funding for centers of excellence from the Parkinson's Foundation and the Lewy Body Dementia Association.

Liana S. Rosenthal has served as an investigator for clinical trials sponsored by Biohaven Pharmaceuticals and the Michael J. Fox Foundation. She serves on the Medical Advisory Board of the National Ataxia Foundation. She receives research support from the $\mathrm{NIH}$, the Michael J. Fox Foundation, the Parkinson's Foundation, the Gordon and Marilyn Macklin Foundation, and the Green Family Foundation.
Marwan Sabbagh is currently serving as an investigator for clinical trials sponsored by $\mathrm{NIH}$. He serves on the Scientific Advisory Board of Bracket. He receives research support from $\mathrm{NIH}$. Dr. Sabbagh has ownership interest in Versanum Inc., Brain Health Inc., Optimal Cognitive Health Company, and uMethod. He consults for Neurotrope, Allergan, Biogen, Bracket, Grifols, vTv Therapeutics, Sanofi, Cortexyme, and Roche-Genentech.

Douglas Scharre has received consulting from BrainTest, royalty fees from BrainTest for the Self-Administered Gerocognitive Examination (SAGE) licensing, and research support from Avanir, Insightec, Merck, Lilly, Axovant, Neurocentria, Astra-Zeneca, Biogen, Novartis, Neurim, Alzheimer's Therapeutic Research Institute (ATRI), Harry T. Mangurian Foundation, Alzheimer's Disease Cooperative Study (ADCS), and Johns Hopkins University.

Sharon Sha receives research support from Biogen Idec, F Hoffmann-La Roche Ltd., Genentech, and Novartis for her role as Investigator in Clinical Trials and has received support from Merck and Alkahest for prior trials. She has been a consultant for Abelson Taylor, Baird, Clearview Healthcare Partners, SelfCare Catalysts Inc., and the University of Southern California. She is funded by NIH grants P50 AG047366 (Stanford Alzheimer's Disease Center) and R01 AG048076-02.

Holly Shill has received research support from Biogen; Dong-A ST Co., Ltd.; MagQu. Intec Pharma, Ltd.; US World Meds; and Sunovion/Cynapsus Therapeutics, Inc., and consulting honoraria for advisory boards from Abbvie and Sunovion

Yasar Torres-Yaghi received consulting fees from Abbvie, Sunovion, and Acorda.

Tina Christie is an employee of the Lewy Body Dementia Association. Todd Graham is an employee of the Lewy Body Dementia Association. lan Richards is an employee of the Lewy Body Dementia Association. Mike Koehler is an employee of the Lewy Body Dementia Association. Brad Boeve has served as an investigator for clinical trials sponsored by GE Healthcare, Axovant, and Biogen. He receives royalties from the publication of a book entitled Behavioral Neurology Of Dementia (Cambridge Medicine, 2009 , 2017). He serves on the Scientific Advisory Board of the Tau Consortium. He receives research support from NIH, the Mayo Clinic Dorothy and Harry T. Mangurian Jr. Lewy Body Dementia Program, and the Little Family Foundation.

\section{Publisher's Note}

Springer Nature remains neutral with regard to jurisdictional claims in published maps and institutional affiliations.

\section{Author details}

${ }^{1}$ Lewy Body Dementia Association, Lilburn, USA. ${ }^{2}$ University of Florida, Gainesville, USA. ${ }^{3}$ University of California, San Diego, USA. ${ }^{4}$ Florida Atlantic University, Boca Raton, USA. ${ }^{5}$ Rush University Medical Center, Chicago, USA. ${ }^{6}$ University of Pennsylvania, Philadelphia, USA. ${ }^{7}$ University of Michigan, Ann Arbor, USA. ${ }^{8}$ University of North Carolina, Chapel Hill, USA. ${ }^{9}$ Cleveland Clinic, Cleveland, USA. ${ }^{10}$ Mayo Clinic campus, 200 1st Street SW, Rochester, MN 55905, USA. ${ }^{11}$ Newcastle University, Newcastle, UK. ${ }^{12}$ University of Rochester, Rochester, USA. ${ }^{13}$ University of Virginia, Charlottesville, USA. ${ }^{14}$ University of Washington, Seattle, USA. ${ }^{15}$ Massachusetts General Hospital, Boston, USA. ${ }^{16}$ Mayo Clinic campus, Jacksonville, FL, USA. ${ }^{17}$ University of Colorado, Aurora, USA. ${ }^{18}$ Columbia University, New York City, USA. ${ }^{19}$ Emory University, Atlanta, USA. ${ }^{20}$ Thomas Jefferson University, Philadelphia, USA. ${ }^{21}$ Georgetown University Medical Center, Washington, D.C., USA. ${ }^{22}$ Johns Hopkins University, Baltimore, USA. ${ }^{23}$ Stanford University, Stanford, USA. ${ }^{24}$ Oregon Health \& Science University, Portland, USA. ${ }^{25}$ Cleveland Clinic Las Vegas, Las Vegas, USA. ${ }^{26}$ The Ohio State University, Columbus, USA. ${ }^{27}$ Barrow Neurological Institute, Phoenix, USA.

\section{Published online: 13 March 2019}

\section{References}

1. McKeith IG, et al. Diagnosis and management of dementia with Lewy bodies: fourth consensus report of the DLB Consortium. Neurology. 2017;89: $88-100$.

2. Thomas AJ, et al. Development of assessment toolkits for improving the diagnosis of the Lewy body dementias: feasibility study within the DIAMOND Lewy study. Int. J. Geriatr. Psychiatry. 2017;32:1280-304. 